\title{
When push comes to shove: exclusion processes with nonlocal consequences
}

\author{
Axel A. Almet, Michael Pan, Barry D. Hughes ${ }^{1, *}$, Kerry A. Landman \\ School of Mathematics and Statistics, University of Melbourne, \\ Victoria 3010 Australia.
}

\begin{abstract}
Stochastic agent-based models are useful for modelling collective movement of biological cells. Lattice-based random walk models of interacting agents where each site can be occupied by at most one agent are called simple exclusion processes. An alternative motility mechanism to simple exclusion is formulated, in which agents are granted more freedom to move under the compromise that interactions are no longer necessarily local. This mechanism is termed shoving. A nonlinear diffusion equation is derived for a single population of shoving agents using mean-field continuum approximations. A continuum model is also derived for a multispecies problem with interacting subpopulations, which either obey the shoving rules or the simple exclusion rules. Numerical solutions of the derived partial differential equations compare well with averaged simulation results for both the single species and multispecies processes in two dimensions, while some issues arise in one dimension for the multispecies case.
\end{abstract}

Keywords:

cell motility, exclusion process, shoving, nonlinear diffusion, multispecies

\footnotetext{
${ }^{*}$ Corresponding author

Email addresses: barrydh@unimelb.edu.au (Barry D. Hughes),

kerryl@unimelb.edu.au (Kerry A. Landman)

${ }^{1}$ Telephone +61383445557, Fax +61383444599
} 


\section{Introduction}

Stochastic agent-based models are useful for modelling complex phenomena such as tumour invasion [1], morphogenic and colonization process during embryonic development [2, 3] and traffic flow [4, 5]. Information can be extracted on two levels: (i) the microscopic level concerning individual agent behaviour through tagging and tracking agents, and (ii) the macroscopic level regarding the collective population behaviour through averaging simulation data over many realizations.

Lattice-based random walk models of interacting agents in which each site is occupied by at most one agent are called exclusion processes $[6,7]$. This restriction on site occupancy emulates a volume-filling constraint, where the spacing between lattice sites is representative of the agent size. There has been much activity in recent years to obtain a continuum-level representation of the macroscopic description of such processes $[8,9,10,11,12]$. Conservation of mass and mean-field arguments relating to the average site occupancy provide the method to transition from probabilistic rules to a continuum model. Unbiased random walkers satisfying a simple exclusion process give rise to a linear diffusion equation, both from rigorous analysis and from mean-field arguments $[6,13]$. No rigorous derivations of associated partial differential equations are available for most interacting agent systems. However, equations produced by mean-field arguments have often been found to give good predictions of the system behaviour averaged over a large number of simulations. Various biologically-motivated mechanisms such as adhesion and myopic movement $[9,11,14,15]$ have been considered. A totally general formulation of mean-field arguments for swarms of monomer agents has been given [16], and there has also been related work on polymeric agents that occupy several lattice sites [17, 18, 19, 20]. For a single species of monomeric agent, all interacting agent models in a very broad class give rise to a nonlinear diffusion equation in a mean-field treatment [16]. However, for the case of multiple species of subpopulations within a total population, the mean-field continuum model for each species is a nonlinear advection-diffusion equation $[8,15,16]$.

A key characteristic of a simple exclusion process is the possibility of aborted moves when an agent attempts to move to an already occupied site. For such an exclusion process, the number of aborted moves increases as the concentration of agents increases. Here we develop a motility mechanism in which attempted moves are always successful, whilst still maintaining the exclusion process. This is achieved through a process termed shoving. This type of movement has been used in previous simulation work on biofilm structures [21, 22, 23], and more generally, biological cells use force to push or shove other cells out of the way [24]. The classic simple exclusion model, where there is no movement into an already occupied site, may therefore not be such a realistic model in various contexts, and the shoving mechanism that we propose allows the draconian simple exclusion restriction to be circumvented. Notions of shoving are also relevant to pedestrian movement, although the way that jostling or shoving is modelled in that context [25] is not as long-ranged as in the model that we shall discuss.

A shoving process may be defined either in continuous time or in discrete time. In continuous time, each agent waits for an exponentially distributed random time before attempting its next move. We shall consider the discrete-time analogue, using random sequential update [5]. If there are $N$ agents present, then at each time, we make $N$ independent random choices of an agent. Each agent chosen is offered an attempt to move before the next agent is chosen. On average, each agent is chosen once per time step. The mean-field analysis that we develop formally corresponds to the limit $N \rightarrow \infty$ and is therefore relevant only when $N \gg 1$.

Under the shoving exclusion process, each agent offered the opportunity to move has a probability $P$ of accepting the offer and attempting a move. The agent attempts to move to one of its $z$ nearest neighbour-sites with equal probability $1 / z$. Like simple exclusion, if an agent attempts to move to an unoccupied site, it will do so. However, if the target site is occupied, the moving agent will shove (push) the agent occupying the target site in the same direction as the moving agent. In this process, the agents do not swap places, but undergo a translation, as highlighted by Fig. 1 for a one-dimensional lattice. All the agents adjacent to the moving agent move in the same direction.

Each agent attempting to move to an occupied site now is certain of shoving the occupant of the target site. This means that interactions between agents are no longer restricted to agents that are adjacent to each other, as illustrated in Fig. 1(b)-(c). The movement of one agent can therefore induce movement of agents some distance away. The mechanism is entirely local in the sense that an agent interacts with an adjacent neighbour in the first instance. However, the consequences are nonlocal and the effects become increasingly significant at larger agent densities, where long lines of adjacent agents are likely to be more prevalent. Yates et al. [26] study forms of pushing mechanisms that are more local in their implications than our model, including pushing only one neighbouring agent into an adjacent vacant site or pushing up to a finite number of agents who occupy adjacent sites in a linear pattern, followed by a vacant site.

Here we study the implications of local movement rules with shoving for the macroscopic description of the population of agents, which may be a single species or multiple species of agents. We shall investigate whether the continuum limit of these probabilistic rules gives rise to a nonlinear diffusion equation and if it does, whether the diffusion coefficient is a polynomial in the average occupancy, as in previous work [9,11, 14, 15]. Alternatively, since our local movement rules have non-local consequences, might one obtain a different form, for example a higher order diffusion equation [27]?

Here we consider three different processes using the shoving mechanism: single species shoving, multispecies shoving and mixed exclusion with one species having the ability to shove while the other does not, which we term "ruffians and gentlemen". 
(a)
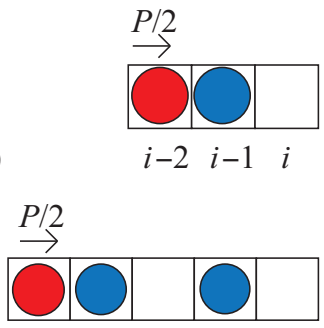

(b) $i-4 \quad i-3 \quad i-2 \quad i-1 \quad i$

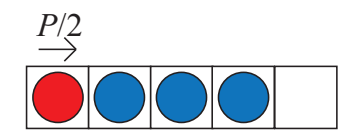

(c) $i-4 \quad i-3 \quad i-2 \quad i-1 \quad i$
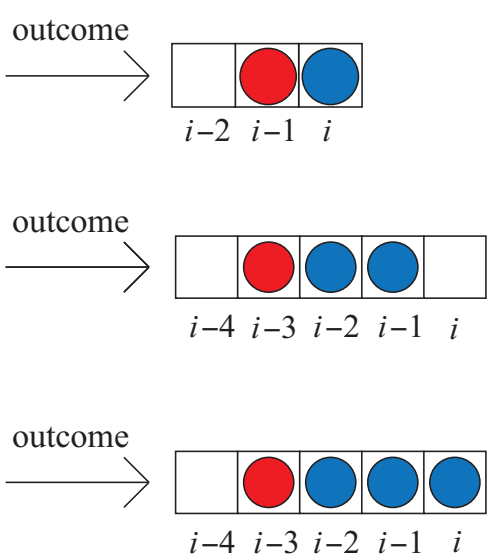

Figure 1: Examples of the shoving process, illustrated on a one-dimensional lattice. The red agent moves with probability $P$, and moves to the right with probability $1 / 2$. (a) The red agent moves into site $i-1$, shoving the blue agent in site $i-1$ to site $i$. (b) The red agent, moving right from $i-4$, will not increase the average occupancy at site $i$ because site $i-2$ is unoccupied. (c) The red agent, moving right from $i-4$, will increase the average occupancy for $i$ because all sites from $i-4$ to $i-1$ are occupied.

\section{Continuum model}

For simplicity, we discuss the one-dimensional lattice-based random walk for the three processes described above and derive a partial differential equation (PDE). The results can be extended naturally to analogous processes on the $d$-dimensional hypercubic lattice $\mathbb{Z}^{d}$, when all shoving that occurs as a result of a given agent move is in the direction of the attempted move. The extension basically involves replacing spatial partial derivatives with gradient or divergence operators. When we proceed to the continuum limit to obtain partial differential equations, the spacing $\Delta$ of lattice sites and the time increment $\tau$ are sent to zero in the usual constrained manner [28] so that

$$
D_{0}=\lim _{\Delta, \tau \rightarrow 0} \frac{P \Delta^{2}}{2 d \tau}
$$

is finite and positive.

The shoving idea should be able to be extended reasonably easily to some periodic lattices other than $\mathbb{Z}^{d}$, such as the twodimensional triangular lattice, but we do not do this here. For lattices which crystallographers would describe as having more than one site per unit cell, such as the face-centred cubic lattice, a somewhat more complicated model would be needed.

It should be noted that we only consider unbounded lattices. For finite lattices, boundary conditions would need to be supplied if agents are placed initially adjacent to the boundary, or if the system has evolved for sufficient time for agents to arrive at the boundary, but we do not address this.

\subsection{Single species}

Consider one population of agents that are all the same type and can either shove or be shoved. We assume that the process takes place on an infinite one-dimensional lattice with spacing $\Delta$. The infinite domain is to avoid any issues of agents being shoved past the boundaries and to guarantee the success of attempted moves. We denote the average occupancy of a site $i$ at time step $n$ by $c_{n}(i)$, where $i \in \mathbb{Z}$ and the time step has length $\tau$. Since site occupancy is either 0 or 1 , the average occupancy $c_{n}(i)$ is a dimensionless number in the interval $[0,1]$. We apply a mean-field approximation that the average occupancy of adjacent sites is independent. Since the shoving process is nonlocal, this independence assumption is applied over many adjacent sites.

A conservation argument gives the change in average occupancy between two time steps:

$$
c_{n+1}(i)-c_{n}(i)=\frac{P}{2}\left[1-c_{n}(i)\right] \sum_{k=1}^{\infty} \prod_{l=1}^{k} c_{n}(i-l)+\frac{P}{2}\left[1-c_{n}(i)\right] \sum_{k=1}^{\infty} \prod_{l=1}^{k} c_{n}(i+l)-\frac{P}{2} c_{n}(i)-\frac{P}{2} c_{n}(i) .
$$

The two positive terms on the right hand side of Eq. (2) represent transitions into the site $i$, provided site $i$ is unoccupied. For example, the product term in the first summation is the result of an agent $k$ sites to the left of site $i$ moving one site to the right resulting an increase in average occupancy in site $i$ (by shoving) if and only if all the sites from $i-1$ to $i-k$ are occupied. This is illustrated in Fig. 1(c) with $k=4$. Since the lattice has no boundary, we must sum all such terms for each integer value of $k \geq 1$. Similarly, the second positive term corresponds to a line of agents extending $k$ sites to the right of site $i$ moving left resulting in an increase in site $i$ occupancy. 
The two negative terms represent transitions out of site $i$. The negative terms are very simple and are the same as for a noninteracting walk, since the move takes place irrespective of whether the sites $i \pm 1$ are vacant or occupied.

It is worth noting that there are other contributions which we have not explicitly written out. The shoving process gives rise to terms resulting from changes in the occupancy at site $i$ when site $i$ is occupied at time step $n$. However equal and opposite terms naturally occur and therefore cancel. In contrast, such terms naturally arise when considering multiple agent species in Section 2.2 , and explicit inclusion of these terms is essential in multispecies cases.

We take the appropriate continuum limit as the distance $\Delta$ and the time $\tau$ between consecutive time steps tend to zero [embodied in Eq. (1) with $d=1$ ] to obtain a PDE description for the average occupancy. On average, each agent will be chosen to move once during each time step $\tau$. We define position $x=\Delta i$ and time $t=n \tau$, and write $c_{n}(i)=C(x, t)$, where $C(x, t)$ is a continuous variable representing the local average occupancy. Supposing $C$ to be sufficiently smooth, we use a Taylor expansion,

$$
c_{n}(i+k)=C+k \Delta \frac{\partial C}{\partial x}+\frac{(k \Delta)^{2}}{2} \frac{\partial^{2} C}{\partial x^{2}}+o\left(\Delta^{2}\right)
$$

where $C$ and its spatial derivatives are evaluated at $(x, t)$. We find that

$$
\sum_{k=1}^{\infty} \prod_{l=1}^{k} c_{n}(i-l)+\sum_{k=1}^{\infty} \prod_{l=1}^{k} c_{n}(i+l)=2 \sum_{k=1}^{\infty} C^{k}+\Delta^{2} \frac{\partial^{2} C}{\partial x^{2}} \sum_{k=1}^{\infty} \sum_{l=1}^{k} l^{2} C^{k-1}+2 \Delta^{2}\left(\frac{\partial C}{\partial x}\right)^{2} \sum_{k=2}^{\infty} \sum_{l=2}^{k} \sum_{j=1}^{l-1} j l C^{k-2}+o\left(\Delta^{2}\right) .
$$

The right hand side can be simplified by judicious interchanges of order of summation and by summation of geometric series and we find that

$$
\left[1-c_{n}(i)\right] \sum_{k=1}^{\infty} \prod_{l=1}^{k} c_{n}(i-l)+\left[1-c_{n}(i)\right] \sum_{k=1}^{\infty} \prod_{l=1}^{k} c_{n}(i+l)=2 C+\Delta^{2} \frac{\partial^{2} C}{\partial x^{2}} \sum_{l=1}^{\infty} l^{2} C^{l-1}+\Delta^{2}\left(\frac{\partial C}{\partial x}\right)^{2} \sum_{l=1}^{\infty} l^{2}(l-1) C^{l-2}+o\left(\Delta^{2}\right) .
$$

The first power series on the right is easily evaluated (by appropriate differentiation of a geometric series) as $(1+C) /(1-C)^{3}$, while the second power series is the derivative of the first power series. Equation (2) can therefore be re-written as

$$
\tau \frac{\partial C}{\partial t}+o(\tau)=\frac{P \Delta^{2}}{2}\left\{\frac{1+C}{(1-C)^{3}} \frac{\partial^{2} C}{\partial x^{2}}+\frac{d}{d C}\left[\frac{1+C}{(1-C)^{3}}\right]\left(\frac{\partial C}{\partial x}\right)^{2}\right\}+o\left(\Delta^{2}\right)
$$

Taking the limit $\Delta, \tau \rightarrow 0$ simultaneously while keeping the ratio $\Delta^{2} / \tau$ constant, a nonlinear differential equation is obtained:

$$
\frac{\partial C}{\partial t}=D_{0} \frac{\partial}{\partial x}\left(\mathcal{D}(C) \frac{\partial C}{\partial x}\right)
$$

where

$$
\mathcal{D}(C)=\frac{1+C}{(1-C)^{3}}
$$

and $D_{0}$ is given by Eq. (1) with $d=1$. Here $\mathcal{D}(C)$ is a rational function which diverges as $C \rightarrow 1$. Some remarks concerning the limit $C \rightarrow 1$ are made in Section 4 .

The arguments above generalize in a straightforward way to $d$-dimensional agent movement, where the direction of the attempted move determines the shoving direction. We denote the standard unit basis vectors generating $\mathbb{Z}^{d}$ by $\left(\hat{\mathbf{e}}_{1}, \hat{\mathbf{e}}_{2}, \ldots, \hat{\mathbf{e}}_{d}\right)$. If we replace $P / 2$ by $P /(2 d), c_{n}(i)$ by $c_{n}(\mathbf{i})$ and $c_{n}(i \pm l)$ by $c_{n}\left(\mathbf{i} \pm l \hat{\mathbf{e}}_{1}\right)$ in the right hand side of Eq. (2), we obtain the expected occupancy change contribution from moves in the first of the $d$ coordinate directions. Adding in the analogous contributions from the remaining coordinate directions and taking the continuum limit leads to the PDE

$$
\frac{\partial C}{\partial t}=D_{0} \nabla \cdot(\mathcal{D}(C) \nabla C)
$$

where $D_{0}$ and $\mathcal{D}(C)$ are given by Eq. (1) and Eq. (7) respectively.

In the dilute system limit $(C \rightarrow 0)$ we find that $\mathcal{D}(C) \rightarrow 1$, that is, linear diffusion is recovered. Also, Eq. (7) gives $\mathcal{D}(C)=$ $1+4 C+O\left(C^{2}\right)$, which may be compared with the exact result that $\mathcal{D}(C)=1+4 C$ when only the shoving of single agents is permitted [26]. A large number of agent-based models with exclusion give rise to polynomial diffusivities $\mathcal{D}(C)$ with $\mathcal{D}(0)=1$ [16], but so far as we are aware, agent-based models leading to diffusivities that are rational functions of $C$ have only been encountered previously for polymeric agents [20]. 


\subsection{Multiple species}

In biological applications it is often convenient to consider subpopulations within a single interacting species [29]. Alternatively, there may be multiple species of populations which interact. Macroscopic models describing the average occupancy of each subpopulation for different agent-based stochastic rules have been investigated $[8,15,16]$. The resulting continuum description is an advection-diffusion equation.

Let us consider multiple species of agents making up the total population. Let $r_{n}(i)$ be the average occupancy of site $i$ at time step $n$ by a single subpopulation (R species), while $c_{n}(i)$ is the average occupancy of site $i$ at time step $n$ of the total population. Suppose that each agent of any of the species has a probability $P$ of attempting a move. By the same arguments as above, we obtain the discrete-time conservation equation

$$
\begin{aligned}
r_{n+1}(i)-r_{n}(i)=\frac{P}{2} & {\left[1-r_{n}(i)\right] r_{n}(i-1)\left\{1+\sum_{k=2}^{\infty} \prod_{l=2}^{k} c_{n}(i-l)\right\}+\frac{P}{2}\left[1-r_{n}(i)\right] r_{n}(i+1)\left\{1+\sum_{k=2}^{\infty} \prod_{l=2}^{k} c_{n}(i+l)\right\} } \\
& -\frac{P}{2} r_{n}(i)\left[c_{n}(i-1)-r_{n}(i-1)\right]\left\{1+\sum_{k=2}^{\infty} \prod_{l=2}^{k} c_{n}(i-l)\right\}-\frac{P}{2} r_{n}(i)\left[c_{n}(i+1)-r_{n}(i+1)\right]\left\{1+\sum_{k=2}^{\infty} \prod_{l=2}^{k} c_{n}(i+l)\right\} \\
& -\frac{P}{2} r_{n}(i)-\frac{P}{2} r_{n}(i),
\end{aligned}
$$

The two positive terms on the right hand side of Eq. (9) represent transitions of $\mathrm{R}$ species into the site $i$, provided site $i$ is unoccupied by an $\mathrm{R}$ agent (that is, either vacant or occupied by another species), but the neighbouring sites $i \pm 1$ are occupied by species R. In the first of these positive terms, the 1 in braces accounts for the event in which the site immediately to the left of site $i$ initiates the move, while the sum over $k$ accounts for the case in which an occupied site $k$ sites to the left of site $i$ initiates the move and all sites between that site and site $i-1$ are occupied (it does not matter by which species they are occupied), so that the $\mathrm{R}$ agent at site $i-1$ is shoved into site $i$.

The first two negative terms on the right hand side of Eq. (9) deal with an R species agent at site $i$ being pushed out by some other species initially adjacent to site $i$, that either initiates the move or is being shoved. The last two negative terms on the right hand side of Eq. (9) simply correspond to the R species agent at site $i$ initiating the move and leaving site $i$ unoccupied. As for the single-species case there are agent moves that do not change the occupancy of site $i$, arising when the agent at $i$ and the agent that displaces it are either both R agents or both agents of another species. Such events do not contribute to the evolution of the R agent occupancy status of site $i$.

In a similar way to the single-species case, we introduce continuous occupancies $R(x, t)$ and $C(x, t)$ of the chosen species and total population respectively, construct Taylor series expansions of these in $\tau$ and $\Delta$ to appropriate order and take the constrained limit $\Delta, \tau \rightarrow 0$ that keeps $\Delta^{2} / \tau$ finite. The algebra is similar to, but more lengthy and intricate than, the algebra for the single-species case and we spare the reader the details. We obtain a nonlinear advection-diffusion equation

$$
\frac{\partial R}{\partial t}=D_{0} \frac{\partial}{\partial x}\left(D(C) \frac{\partial R}{\partial x}+R V(C) \frac{\partial C}{\partial x}\right)
$$

where

$$
D(C)=\frac{1}{1-C}, V(C)=\frac{3-C}{(1-C)^{3}},
$$

and $D_{0}$ is given by Eq. (1) with $d=1$. We now see that the diffusivity and the advection coefficient both diverge as $C \rightarrow 1$, but the advection term diverges more rapidly. Each subspecies satisfies the same type of PDE.

The arguments above can be extended to $d$-dimensional agent movement, where the direction of the attempted move determines the shoving direction. The resulting PDE is

$$
\frac{\partial R}{\partial t}=D_{0} \nabla \cdot(D(C) \nabla R+R V(C) \nabla C)
$$

where $D(C), V(C)$ and $D_{0}$ are given by Eq. (11) and Eq. (1) respectively.

When considering the total population, where all the subpopulations are identical, the conservation equations for each species can be summed to produce a single equation for the total population $C$. The resulting equation is the nonlinear diffusion equation appropriate to a single species, namely Eq. (8), when we identify

$$
\mathcal{D}(C)=D(C)+C V(C)
$$

This is the same identification as found for other interaction rules in multispecies systems [16], where shoving is not allowed. 


\subsection{Mixed processes: ruffians and gentlemen}

We now consider the interaction of two populations that behave according to different mechanisms. One population (R agents) is always able to move actively (to a vacant site or through the shoving process if the site is occupied), while the second population ( $\mathrm{G}$ agents) can only initiate a move if adjacent sites are empty, but they can be shoved by a movement of the first population (and if shoved will shove others of either species). We call this the ruffians and gentlemen model. The R agents ("ruffians" or red agents) act as bullies: if they are selected to move, they can shove an adjacent agent of either species or a chain of agents of any composition. The G agents ("gentlemen" or green agents) are well-mannered, and for them an attempt to move actively onto an occupied site (whether occupied by an R agent or a $\mathrm{G}$ agent) is always aborted.

We denote the average occupancy at time $n$ of site $i$ by $\mathrm{R}$ agents or by $\mathrm{G}$ agents by $r_{n}(i)$ and $g_{n}(i)$, respectively. The average occupancy, irrespective of species, is $c_{n}(i)=r_{n}(i)+g_{n}(i)$. The change in the average occupancy $r_{n}(i)$ of site $i$ by an R agent in one time step can be obtained in terms of the average occupancy of the $r_{n}(i), g_{n}(i)$ and the total occupancy $c_{n}(i)=r_{n}(i)+g_{n}(i)$. If we adopt the usual convention that a product for which the lower terminal exceeds the upper terminal is interpreted as taking the value 1 , then the change in the average occupancy $r_{n}(i)$ of site $i$ by an R agent at the $n$th time step is

$$
\begin{aligned}
r_{n+1}(i)-r_{n}(i)= & \frac{P}{2} r_{n}(i-1)\left[1-r_{n}(i)\right]\left[1+\sum_{k=2}^{\infty} r_{n}(i-k) \prod_{l=2}^{k-1} c_{n}(i-l)\right]+\frac{P}{2} r_{n}(i+1)\left[1-r_{n}(i)\right]\left[1+\sum_{k=2}^{\infty} r_{n}(i+k) \prod_{l=2}^{k-1} c_{n}(i+l)\right] \\
& -\frac{P}{2} g_{n}(i-1) r_{n}(i) \sum_{k=2}^{\infty} r_{n}(i-k) \prod_{l=2}^{k-1} c_{n}(i-l)-\frac{P}{2} g_{n}(i+1) r_{n}(i) \sum_{k=2}^{\infty} r_{n}(i+k) \prod_{l=2}^{k-1} c_{n}(i+l)-\frac{P}{2} r_{n}(i)-\frac{P}{2} r_{n}(i) .
\end{aligned}
$$

The first term covers the case in which site $i$ does not contain an $\mathrm{R}$ agent, but becomes occupied by an $\mathrm{R}$ agent either because an $\mathrm{R}$ agent lies immediately to its left and chooses to move to site $i$, or because an $\mathrm{R}$ agent occupies site $i-1$ and an $\mathrm{R}$ agent at site $i-k$ moves right and pushes an unbroken chain of agents found between itself and site $i-1$. The second term has an analogous interpretation involving sites moving left. The third term of the right hand side of (14) describes agents $k$ sites to the left of site $i$ moving to the right, decreasing the average occupancy by shoving if and only if an $\mathrm{R}$ agent is occupying site $i-k$ and a $\mathrm{G}$ agent is occupying site $i-1$ with all sites in between being occupied, provided an $\mathrm{R}$ agent is in site $i$. Similar reasoning is given for the fourth term. The last two terms describe an $\mathrm{R}$ agent in site $i$ initiating a move left or right, leaving site $i$ vacant.

As for the single species, we take the Taylor series in $\Delta$ and $\tau$. For continuous time and space occupancy $R(x, t)$ for the $\mathrm{R}$ species (ruffians) and total population occupancy $C(x, t)$, and taking the appropriate limit $\Delta, \tau \rightarrow 0$, we obtain a nonlinear advection-diffusion equation

$$
\frac{\partial R}{\partial t}=D_{0} \frac{\partial}{\partial x}\left(D(R, C) \frac{\partial R}{\partial x}+R V(R, C) \frac{\partial C}{\partial x}\right),
$$

where

$$
D(R, C)=1+2 R \frac{2-C}{(1-C)^{2}}, V(R, C)=R \frac{3-C}{(1-C)^{3}},
$$

and $D_{0}$ given in Eq. (1) with $d=1$. We see again that the diffusivity and the advection coefficient both diverge as $C \rightarrow 1$, but the diffusion term diverges more rapidly than in the simpler multispecies case where all species behave as ruffians [see Eq. (11)]. These formulae can be checked for the limiting case when there are no $\mathrm{G}$ agents. For this case, $R=C$ and $\operatorname{since} D(C, C)+C V(C, C)=$ $(1+C) /(1-C)^{3}=\mathcal{D}(C)$, the PDE reduces to Eq. (7), as expected.

For the $\mathrm{G}$ species (gentlemen), we must account for a change in the average occupancy due to simple exclusion, and the change in average occupancy due to shoving from movements initiated by ruffian R species. Noting again that products for which the lower terminal exceeds the upper terminal are interpreted as unity, the change in average occupancy of $g_{n}(i)$ is given by

$$
\begin{aligned}
g_{n+1}(i)-g_{n}(i)=\frac{P}{2} & g_{n}(i-1)\left[1-c_{n}(i)\right]+\frac{P}{2} g_{n}(i+1)\left[1-c_{n}(i)\right]-\frac{P}{2} g_{n}(i)\left[1-c_{n}(i-1)\right]-\frac{P}{2} g_{n}(i)\left[1-c_{n}(i+1)\right] \\
& +\frac{P}{2} g_{n}(i-1)\left[1-g_{n}(i)\right] \sum_{k=2}^{\infty} r_{n}(i-k) \prod_{l=2}^{k-1} c_{n}(i-l)+\frac{P}{2} g_{n}(i+1)\left[1-g_{n}(i)\right] \sum_{k=2}^{\infty} r_{n}(i+k) \prod_{l=2}^{k-1} c_{n}(i+l) \\
& -\frac{P}{2} r_{n}(i-1) g_{n}(i)\left[1+\sum_{k=2}^{\infty} r_{n}(i-k) \prod_{l=2}^{k-1} c_{n}(i-l)\right]-\frac{P}{2} r_{n}(i+1) g_{n}(i)\left[1+\sum_{k=2}^{\infty} r_{n}(i+k) \prod_{l=2}^{k-1} c_{n}(i+l)\right] .
\end{aligned}
$$

The identification of each term is similar to previous interpretations, and so is not repeated. Taking Taylor series and the usual continuum limits, we obtain

$$
\frac{\partial G}{\partial t}=D_{0} \frac{\partial}{\partial x}\left\{\left(1-C+\frac{R}{1-C}\right) \frac{\partial G}{\partial x}+G\left[\frac{3-C}{(1-C)^{2}} \frac{\partial R}{\partial x}+\left(1+\frac{R(3-C)}{(1-C)^{3}}\right) \frac{\partial C}{\partial x}\right]\right\}
$$



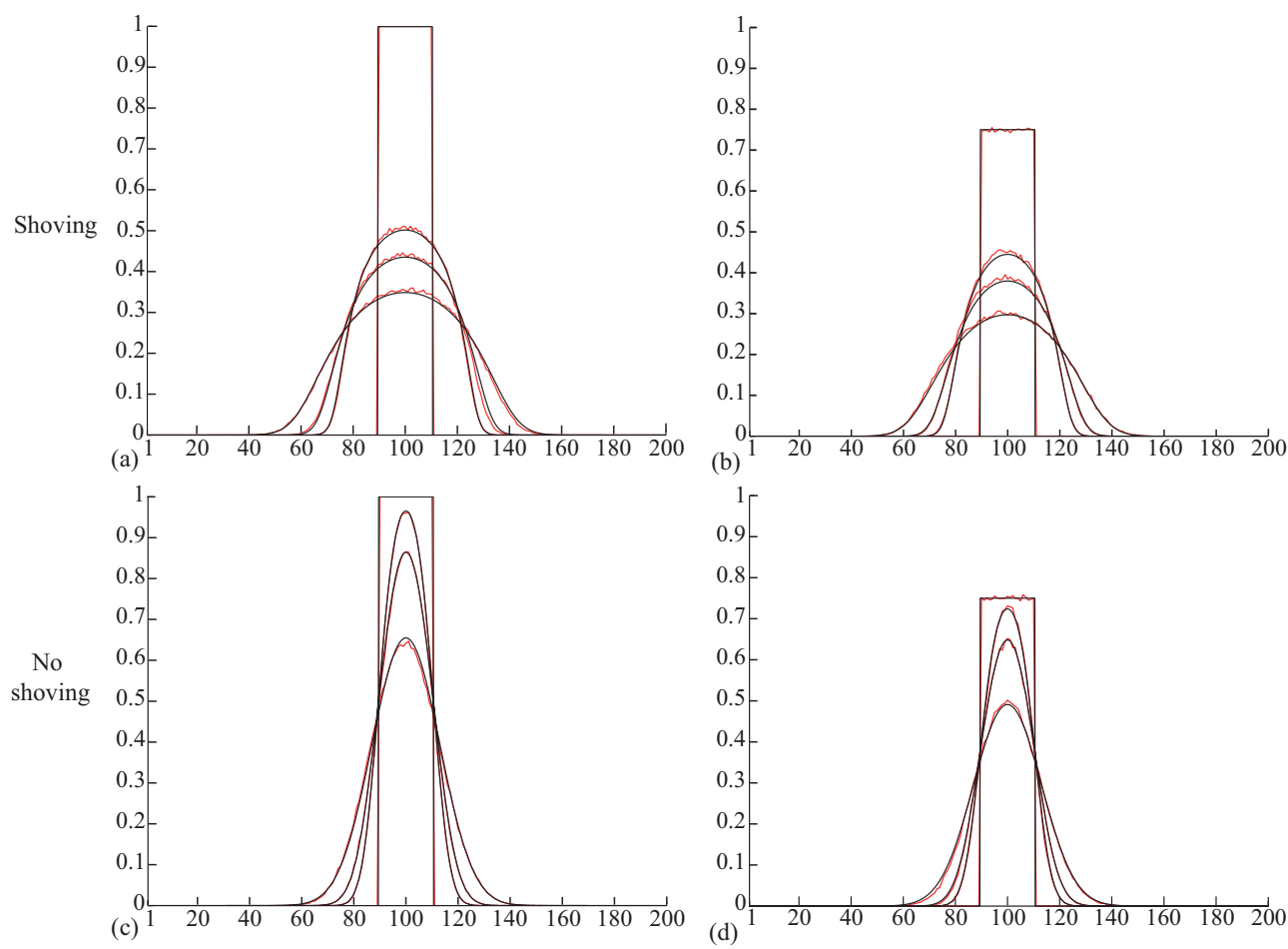

Figure 2: Single species averaged column density data (red) compared with PDE solutions to the PDE (black) at times $t=0,50,100,250$. Agents spread from $90 \leq x \leq 110$ on a $200 \times 20$ lattice, for two initial conditions and the results are averaged over 750 realizations. (a-b) Shoving rules and PDE Eq. (8) with initial density (a) 0.999 and (b) 0.75 . (c-d) In the absence of shoving and only simple exclusion, the linear diffusion PDE solution with initial density (c) 0.999 and (d) 0.75 .

Note that if $R=0$ this reduces to the linear diffusion equation for $G$, as expected, since only a simple exclusion process is occurring. The PDE governing the G subpopulation is also a nonlinear advection-diffusion equation. Of course, this PDE could be written in an alternative form if we eliminate $R$ using $R=C-G$ to give

$$
\frac{\partial G}{\partial t}=D_{0} \frac{\partial}{\partial x}\left\{\left(1-G+\frac{C^{2}(1-C)+G\left(C^{2}-3\right)}{(1-C)^{2}}\right) \frac{\partial G}{\partial x}+G\left(1+\frac{(1-G)(3-C)}{(1-C)^{3}}\right) \frac{\partial C}{\partial x}\right\} .
$$

Equations (15) and (19) can be extended to $d$ dimensions in the obvious way.

\section{Simulation and results}

To test the validity of the mean-field arguments, we generate and compare results from the discrete and continuum models for single, multiple species, and ruffians and gentlemen models.

We consider a two-dimensional square lattice, with spacing $\Delta=1$ and we set the duration of a time step to be $\tau=1$. Although the mean-field PDE is obtained in the limit $\Delta \rightarrow 0$ and $\tau \rightarrow 0$, experience with previous studies of agent-based simulations has shown that the match between simulations and PDEs does not require $\Delta$ and $\tau$ to be particularly small [8, 14], but only small compared to the overall system size and the duration of the simulation, respectively. We work with a lattice fragment of size $200 \times 20$ with periodic boundary conditions imposed on the horizontal boundaries and reflecting boundary conditions (no flux) imposed on the vertical boundaries at $x=0$ and $x=200$. An initial agent density is prescribed within the strip $90 \leq x \leq 110$. Note that here the horizontal length is sufficiently large that there is negligible probability that any agents will encounter the vertical boundaries for $t \leq 250$, the maximum duration of the simulations that we consider. (Column-averaged density data is averaged over 750 identically prepared realizations. The probability of attempted movement by each agent offered the chance to move is $P=1$.)

Since the initial conditions are independent of the vertical coordinate, the nonlinear diffusion and advection-diffusion equations reduce to PDEs with spatial derivatives in a single $x$ variable. Note however that as the underlying PDEs are two-dimensional, the value of $D_{0}$ in Eqn. (1) uses $d=2$. These PDEs are solved numerically using Matlab pdepe (which calls the stiff solver ode15s, a multistep method with adaptive time stepping) with a finite difference method with constant grid spacing $\delta x=0.1$. The results were checked to be grid-independent. 


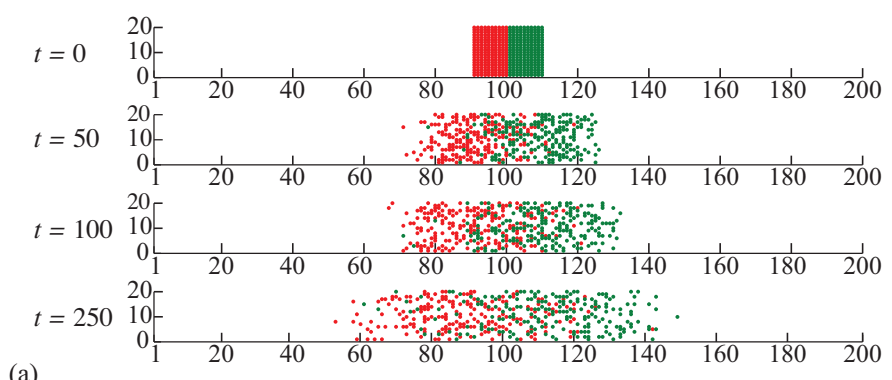

(a)
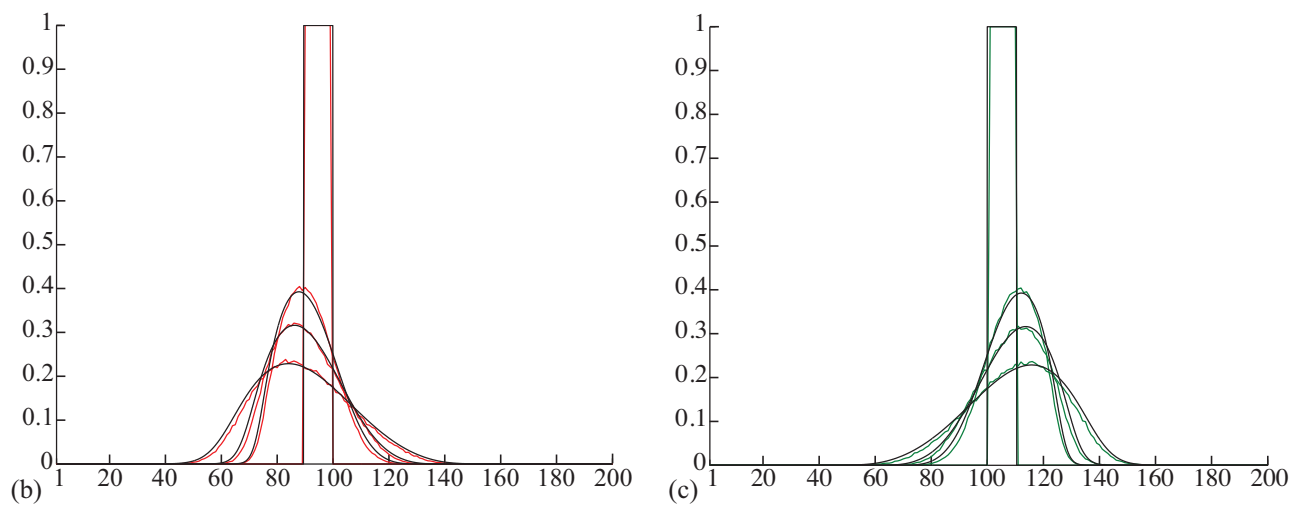

Figure 3: Multiple species in two dimensions at times $t=0,50,100,250$. Agents spread from $90 \leq x \leq 110$ on a $200 \times 20$ lattice. (a) A single simulation. (b-c) Averaged column density data (red and green) compared with PDE solutions to the PDE (black). Simulations are averaged over 750 realizations.

For every realization at $t=0$, the middle sites $90 \leq x \leq 110$ were filled randomly with the probability of a site being occupied being either 0.999 or 0.75 . The initial occupancy of 0.999 was used since we cannot solve the PDE with full occupancy, due to $C=1$ being a singular point of the diffusion coefficient.

Comparison of the solution of Eq. (8) with column-averaged density profiles from the simulations in Fig. 2 shows an excellent correspondence. The shoving process allows the agents to spread faster than the simple exclusion process, due to fewer aborted moves in the simulation. We checked results on one-dimensional lattices as well and the comparison between the continuum and discrete data is of comparable quality to the ones shown here. Furthermore, we have also run some two-dimensional simulations in which the agents are all initially located in a compact region of a large lattice, such as the closest lattice equivalent to a circular disc. We find that the effects of shoving decay rapidly with time as the initial cluster breaks up into small fragments. For this initial condition, the agent population remains in an approximately symmetrical circular distribution, so that there are no long-lasting lattice-induced anisotropies caused by the shoving mechanism.

Now consider two distinguishable but otherwise identical species of agents (red and green, respectively). Initially the sites $90 \leq x \leq 99$ were filled with red agents and sites $100 \leq x \leq 109$ were filled with green agents, where the filling probability was 0.999 (as described above). Figure 3 shows single simulation results, as well as column averaged results. The comparison between the PDE solutions and the simulations is excellent. Note that in two dimensions, the red and green agents mix as observed in Fig. 3(a), since they can move around each other through vertical movements. However, this is not the case in one dimension (Fig. 4(a)). This constraint is the likely reason that the PDE does not match the data well in one dimension as illustrated in Fig. 4(b)(c). For this case, the mean-field approximation is not valid, since the location of red and green agents is partially determined by their initial position.

To check this interpretation of the reason for the mismatch in one dimension between the PDE solution and simulation average occupancies, we have repeated the one-dimensional simulations with an alternative initial condition. Instead of starting with adjacent fully occupied intervals, with the left interval fully occupied by agents of one species and the right interval fully occupied by agents of the other species, we give each site in the interval $90 \leq x \leq 110$ a probability 0.4 of being occupied initially by a green agent, a probability 0.4 of being initially occupied by a red agent, and a probability 0.2 of being unoccupied. We find that the average occupancies of each species now match the PDE solutions very closely.

Finally we consider the case when a population consists of ruffians (red) and gentlemen (green), shown in Fig. 5. As time evolves we see the gentlemen agent profile increases in skewness, while the ruffian profile remains much less asymmetric. The match between the results ofsimulations and the solutions of the coupled continuum equations given by Eqs. (15), (16) and (18) is again excellent in two dimensions for an initial occupancy of 0.75 for both populations. The match in one dimension is poor again due to the absence of mixing between the two populations.

Except for one-dimensional multispecies cases with species-segregated initial conditions, the match between mean-field PDE 

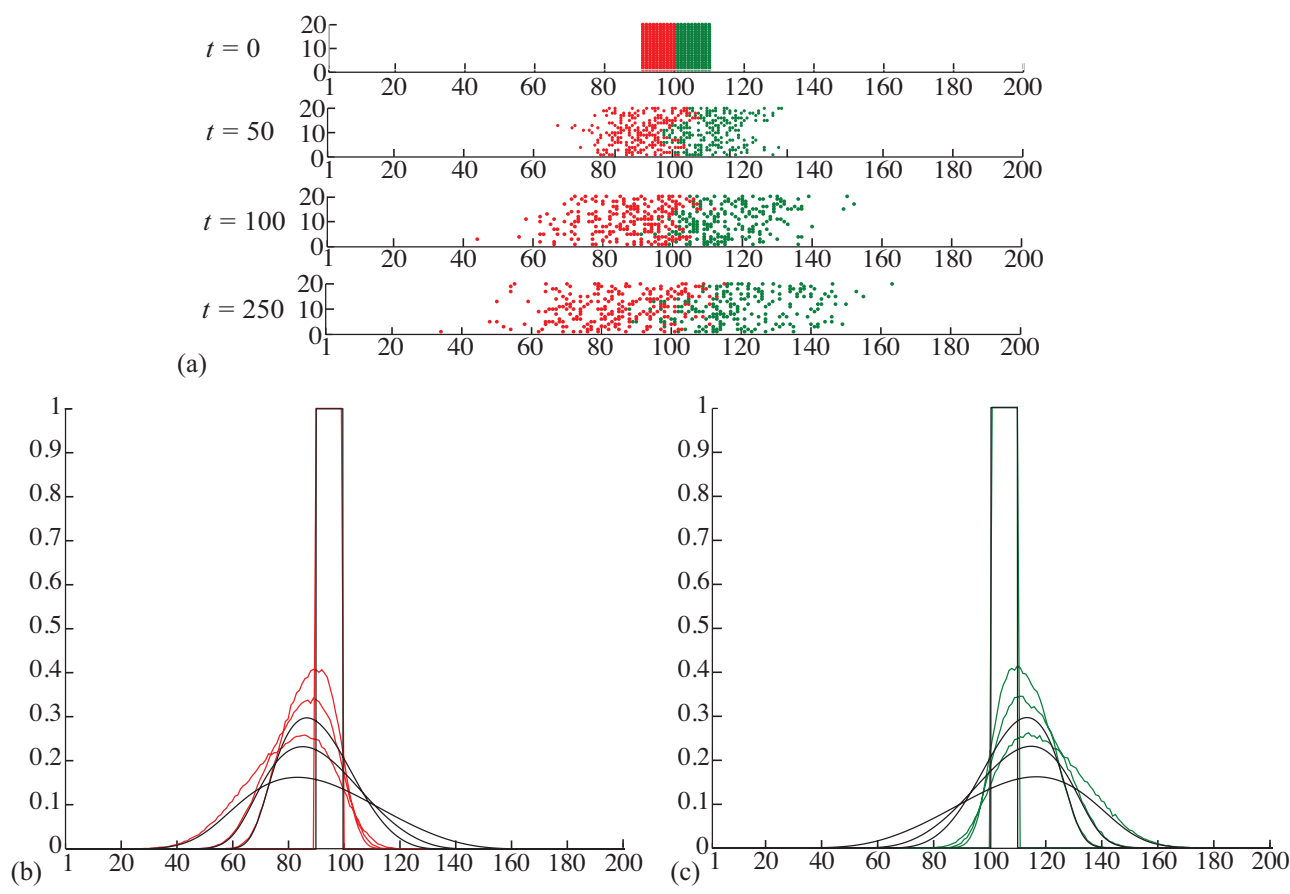

Figure 4: Multiple species in one dimension at times $t=0,50,100,250$. Agents spread from $90 \leq x \leq 110$ on a $200 \times 20$ lattice. (a) 20 simulations are shown. (b-c) Averaged column density data (red and green) compared with PDE solutions to the PDE (black). For fair comparison against our results for two-dimensional simulations (which used 750 realizations of a 20-row system, column-averaged), these one-dimensional simulations are averaged over 15000 realizations.

solutions and average occupancies found by simulation is very good. In principle, superior matching may be possible if account is taken of correlations, perhaps by finding approximate evolution equations for pair correlation functions [30], but the resulting formalism requires the solution of a large number of ordinary differential equations (several for each lattice site) rather than the solution of PDEs, and the physical interpretation in terms of diffusivities and advective velocities is obscured.

\section{Discussion}

We have considered an alternative model to the well-known and frequently used exclusion process. This alternative model involves indiscriminate shoving in order to facilitate movement. Unlike the simple exclusion process, there are no aborted moves for those agents who are permitted to shove other agents. Furthermore, any movement of an agent no longer just has local consequences, but now can have global consequences, if the moving agent has many neighbours.

Using mean-field theory, PDEs have been derived to describe the average behaviour of three implementations of the shoving process: a single-species model, a multispecies model in which all species are allowed to shove, and a two-species model in which one species can shove but the other cannot ("ruffians and gentlemen"). Comparisons of the PDE solutions with averaged simulation results in two dimensions have confirmed that the PDE solutions are good predictors of the average occupancy for this discrete motility mechanism for all the three cases considered, namely single species, multiple species and mismatched multiple species. In one dimension, since agents do not have the capacity to pass each other, the mean-field approximation is always good for a single species, but breaks down for multiple species for segregated initial conditions, thereby giving a mismatch between PDE solutions and simulation results.

The PDEs obtained are of the same type as previously found for various interacting species, namely nonlinear diffusion equations for single species equations and advection-diffusion equations for multiple species systems [16]. Until recently, the continuum limits of symmetric probabilistic rules have yielded the diffusivity and advection functions to be polynomial functions [16]. However, in a more advanced treatment of elongated agents (agents which occupy more than a single lattice site), the diffusivity was also found to be a rational function, which arose from the more complex form of the mean-field approximation needed for elongated agents [20]. Also, these diffusivities were non-singular over the possible ranges of the average occupancy variable. In contrast, we have found here that the diffusivity and advection functions are singular functions which diverge as the occupancy reaches the maximum value of unity.

Our observations regarding the shape of the diffusion coefficient and their values at maximum density should not be overinterpreted since the agent movement depends on the flux which is a product of two terms:

$$
J=-\mathcal{D}(C) \nabla C .
$$



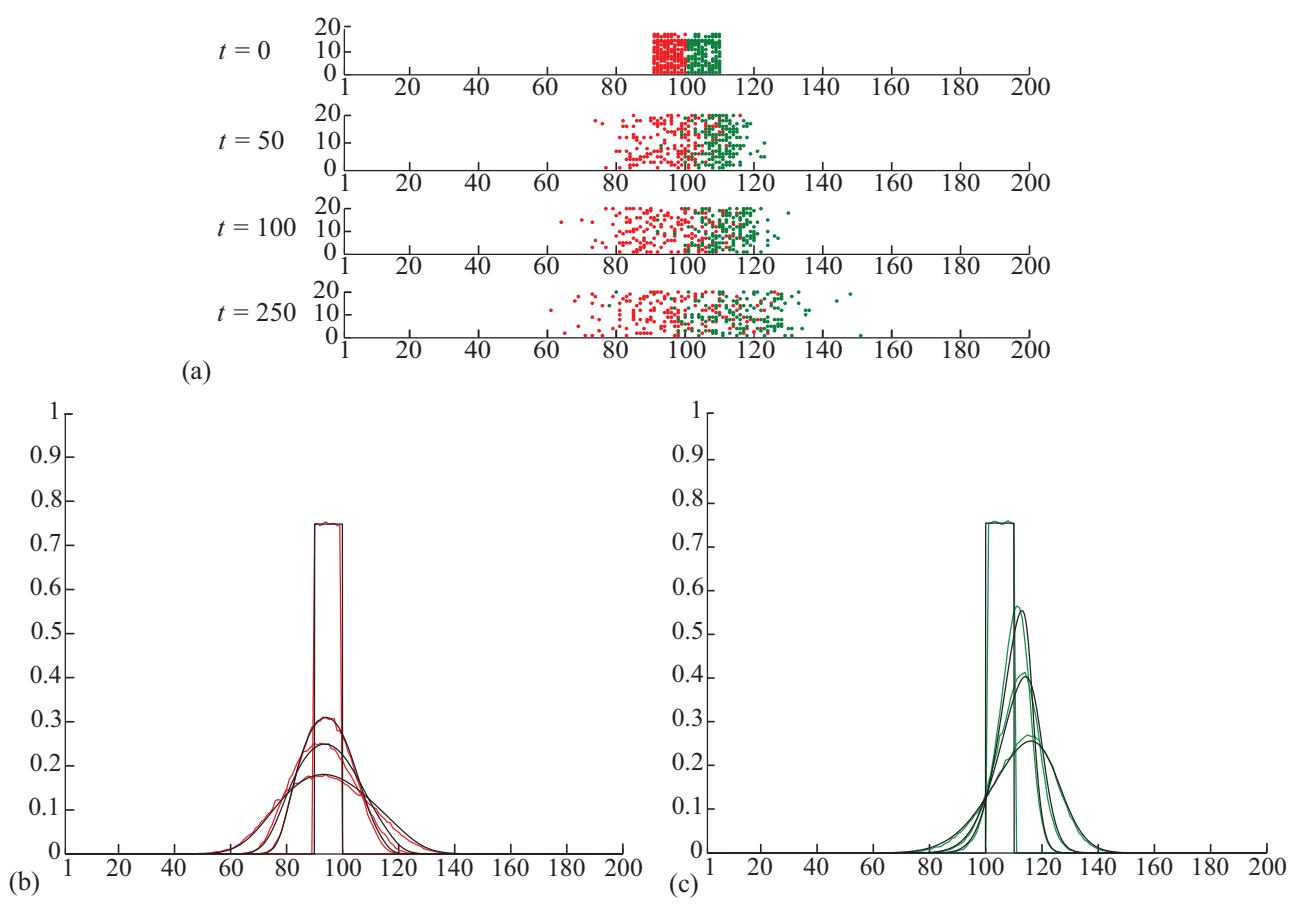

Figure 5: Ruffians and gentlemen exclusion process in two dimensions at times $t=0,50,100,250$ with initial occupancy of 0.75 . Agents spread from $90 \leq x \leq 110$ on a $200 \times 20$ lattice. (a) A single simulation with ruffians (red) on the left and gentlemen (green) on the right. (b-c) Averaged column density data (b) ruffians (red) and (c) gentlemen (green) compared with PDE solutions to the PDE (black). Simulations are averaged over 750 realizations.

Indeed, at the maximum density the gradient in the local agent occupancy will be zero. It is only necessary that $J$ is defined everywhere.

Furthermore, the difference between self-diffusion of a single tagged agent in a collection of similarly moving agents and the population-level diffusivity is critically important. For example, consider the case of simple exclusion with no shoving [15]. The mean-squared displacement of a single agent in a simple exclusion process is proportional to $D_{0}(1-C)$, so decreases linearly with $C$ due to crowding. However, when the continuum limit of the master equation is taken, the corresponding PDE has a diffusive flux term namely $-D_{0}(1-C) \nabla C$ and a convective flux term proportional to $-D_{0} C \nabla C$. The two together cancel the nonlinear term, producing net flux of $-D_{0} \nabla C$, thus producing a linear diffusion equation. This demonstrates that the intuitive expectation that the diffusivity decreases with crowding is false, as it remains a constant in the most simple case.

Here we have posed and addressed the first treatment of a simple shoving process with long-range shoving permitted, for single and multiple species. As noted earlier, Yates et al. [26] consider variants of a single-species model in which a milder form of shoving is permitted. Several extensions are possible. Instead of allowing all the agents adjacent to the moving agent to move in the same direction, one could consider the more complex process where movements can be in any direction. For example, on a square lattice in two dimensions, an agent moving south may split a group of horizontally aligned neighbouring agents so that some move west or east to accommodate for the agent in their alignment. This is a perpendicular shove. This mechanism may be the dominant one responsible for the ovarian cancer cells pushing the mesothelial cells out of the way in Iwanicki et al. [24]. In this first model, any agent can push any number of agents one lattice spacing. In practice, there will be resistance to shoving, and there may be a maximum number of agents that can be shoved at any one time. Alternatively, we could address this by introducing a shove weighting which is a function of the number of agents that require shoving, resulting in shoves of a high number of agents being less successful than shoves of a small number of agents. These extensions may remove the singularity in the diffusion coefficients.

Of course, an alternative to shoving as a process for ensuring that agent moves are always successful in an exclusion process is to include agents swapping (exchanging) sites. However, such a rule has only local consequences and is not discussed here. As we noted earlier, although our interactions between agents are locally mediated, the consequences are nonlocal. From the nature of the local interactions, when mean-field approximations are used and the continuum limit is taken, second-order PDEs of parabolic type are obtained. We draw a distinction between our work and other forms of agent-based modelling where some form of nonlocality is embodied in PDEs of higher order, or convolution integral contributions to evolution equations [27, 31, 32, 33].

We have not allowed agents in our model to proliferate: we have only been concerned with questions of motility. In simulation, the addition of proliferation would be straightforward. Although in cellular biology, proliferation corresponds to cell division, if for clarity in specifying mechanisms one speaks of the mother and the baby, one could consider either allowing proliferation only when a site adjacent to the mother is available for the baby to be deposited, or allowing the mother to shove the baby [26]. For 
simple exclusion with proliferation, the partial differential equations that arise in the continuum limit from a mean-field analysis reproduce average behaviour obtained from simulations only for suitably low proliferation rates. The problem is essentially one of correlation in site occupancy. It would be interesting to study the effect of proliferation on our long-range shoving model.

Agent-based models in computational biology and biological physics and the study of their emergent properties and patterns is of great interest. Determining the average population-level results of biologically motivated agent rules is an important step in that understanding.

\section{Acknowledgments}

This work was supported by the Australian Research Council (DP1110100795 and DP140100339) and an Australian Mathematical Sciences Institute student vacation scholarship (A.A. Almet). We thank an anonymous reviewer for alerting us to the manuscript [26].

\section{References}

[1] L.M. Sander, T.S. Deisboeck, Phys. Rev. E 66 (2002) 051901.

[2] B.C. Thorne, A.M. Bailey, D.W. DeSimone, S.M. Peirce, Birth Defects Res. C 81 (2007) 344-353.

[3] B.L.Cheeseman, D. Zhang, B.J. Binder, D.F. Newgreen, K.A. Landman, J Royal Soc. Int. 11 (2014) 20130815.

[4] A. Schadschneider, Physica A 313 (2002) 153-187.

[5] D. Chowdhury, A. Schadschneider, K. Nishinari, Phys. Life Rev. 2 (2005) 318-352.

[6] T.M. Liggett, Stochastic Interacting Systems: Contact, Voter and Exclusion Processes, Springer-Verlag, Berlin, 1999.

[7] F. Spitzer, Adv. Math. 5 (1970) 256-290.

[8] M.J. Simpson, K.A. Landman, B.D. Hughes, Physica A 388 (2009) 399-406.

[9] C. Deroulers, M. Aubert, M. Badoual, B. Grammaticos, Phys. Rev. E 79 (2009) 031917.

[10] D. Fanelli, A.J. McKane, Phys. Rev. E 82 (2010) 021113.

[11] A.E. Fernando, K.A.Landman, M.J. Simpson, Phys. Rev. E 81(2010) 011903.

[12] K.J. Painter, Bull. Math. Biol. 71 (2009) 1117-1147.

[13] M.J. Simpson, K.A. Landman, B.D. Hughes, Aust. J. Eng. Ed. 15 (2009) 59-67.

[14] M.J. Simpson, K.A. Landman, B.D. Hughes, A.E. Fernando, Physica A 389 (2010) 1412-1424.

[15] K.A. Landman, A.E. Fernando, Physica A 390 (2011) 3742-3753.

[16] C.J. Penington, B.D. Hughes, K.A. Landman, Phys. Rev E 84 (2011) 041120.

[17] M.J. Simpson, R.E. Baker, S.W. McCue, Phys. Rev. E 83 (2011) 021901.

[18] R.E. Baker, M.J. Simpson, Physica A 391 (2012) 3729-3750.

[19] C.J. Penington, K. Korvasová, B.D. Hughes, K.A. Landman, Phys. Rev. E 86 (2012) 051909.

[20] C.J. Penington, B.D. Hughes, K.A. Landman, Phys. Rev. E 89 (2014) 032714.

[21] C.S. Laspidou, B.E. Rittmann, Water Res. 38 (2004) 3349-3361.

[22] C.S. Laspidou, B.E. Rittmann, Water Res. 38 (2004) 3362-3371.

[23] C.S. Laspidou, A. Kungolosb, P. Samarasc, Desalination 250 (2010) 390-394.

[24] M.P. Iwanicki, R.A. Davidowitz, M.R. Ng, A. Besser, T. Muranen, M. Merritt, G. Danuser, T. A. Ince, J.S. Brugge, Cancer Discovery J. 1 (2011) $144-57$.

[25] T.J. Lightfoot, G. Milne, The Australian Conference on Artificial Life (ACAL), (2003) 159-169 (http://smr.csse.uwa.edu.au/pdf/Modelling_ Emergent_Crowd_Behaviour.pdf).

[26] C.A. Yates, A. Parker, R.E. Baker, preprint, http://biorxiv.org/content/biorxiv/early/2014/12/09/012492.full.pdf; and supplementary material, http://biorxiv.org/content/biorxiv/suppl/2014/12/09/012492.DC1/012492-1.pdf

[27] J.D. Murray, Mathematical Biology, Vol. 1, 3rd edition, Springer, New York, 2002.

[28] B.D. Hughes, Random Walks and Random Environments, Vol. 1, Oxford University Press, 1995.

[29] M.J. Simpson, D.C. Zhang, M. Mariani, K.A. Landman, D.F. Newgreen, Dev. Biol. 302 (2007) $553-568$.

[30] D.C. Markham, M.J. Simpson, R.E. Baker, Phys. Rev. E 87 (2013) 062702.

[31] N.J. Armstrong, K.J. Painter, J. A. Sherratt, J.Theor. Biol. 243 (2006) 98-113.

[32] E.J. Hackett-Jones, K.A. Landman, K. Fellner, Phys. Rev. E 85 (2012) 041912.

[33] B.D. Hughes, K. Fellner, Physica D 260 (2013) 26-48. 


\section{University Library}

\section{- M M N E R VA A gateway to Melbourne's research publications}

Minerva Access is the Institutional Repository of The University of Melbourne

Author/s:

Almet, AA;Pan, M;Hughes, BD;Landman, KA

Title:

When push comes to shove: Exclusion processes with nonlocal consequences

Date:

2015-11-01

Citation:

Almet, A. A., Pan, M., Hughes, B. D. \& Landman, K. A. (2015). When push comes to shove: Exclusion processes with nonlocal consequences. PHYSICA A-STATISTICAL MECHANICS AND ITS APPLICATIONS, 437, pp.119-129. https://doi.org/10.1016/j.physa.2015.05.031.

Persistent Link:

http://hdl.handle.net/11343/58827 